\title{
Control of ring rolling with variable thickness and curvature
}

\author{
Matthew R. Arthington ${ }^{1}$. Jos Havinga ${ }^{1,2}$ (D) . Stephen R. Duncan ${ }^{1}$
}

Received: 9 November 2018 / Accepted: 16 January 2019 / Published online: 6 February 2019

(c) The Author(s) 2019

\begin{abstract}
Radial-Axial Ring Rolling (RARR) is an industrial forging process for making strong, seamless metal rings. Conventionally, rings are made circular with constant cross-section. In this work we demonstrate a sensing and control strategy to create rings with variable radial wall thickness and variable curvature using standard RARR hardware. This has a number of potentially useful applications but also provides an understanding of how to control these properties for conventional RARR. The sensing uses a calibrated video camera to take a series of images of the ring top surface. Image processing is employed to measure and track the ring material in-situ. The complete state of the ring is represented by the ring thickness and curvature as a function of its volume fraction, which is computed by combining the measurements from the unoccluded areas with estimates of the ring shape elsewhere. Additionally, we present a marking technique for tracking of material as it rotates through the rolling machine, even after significant deformation of the ring has occurred. We show that rings with a wide range of variation in local thickness and curvature can be formed using conventional RARR hardware and a photogrammetric state measurement technique, combined with open-loop scheduling and feedback control of thickness and curvature. Rings with both variable thickness and non-circular shapes have been produced virtually using numerical simulations and in reality using modelling clay as a material to simulate metals at forging temperatures. We demonstrate that this technique extends the range of shapes achievable with standard RARR hardware.
\end{abstract}

Keywords Process automation · Process control $\cdot$ Digital image correlation $\cdot$ Industrial control $\cdot$ Ring rolling ·

Variable geometry

\section{Introduction}

Radial-Axial Ring Rolling (RARR) is an industrial hot forging process that produces seamless metal rings with

This work was funded by the Engineering and Physical Sciences Research Council (EPSRC) UK (project reference $\mathrm{EP} / \mathrm{K} 018108 / 1$ ).

The authors are grateful for the contributions made by Chris Cleaver and Jiangling Huang towards this work.

Stephen R. Duncan

stephen.duncan@eng.ox.ac.uk

Matthew R. Arthington

matthew.arthington@pmb.ox.ac.uk

Jos Havinga

jos.havinga@utwente.nl

1 Department of Engineering Science, University of Oxford, 17 Parks Road, Oxford, OX1 3PJ, UK

2 Faculty of Engineering Technology, University of Twente, Drienerlolaan 5, 7522NB, Enschede, The Netherlands desirable material properties [11]. Figure 1 shows the configuration of a conventional machine:

- the mandrel compresses the ring wall radially against the forming roll in the radial pass, and the forming roll is driven to draw the ring into the roll gap using friction whilst the mandrel rotates idly;

- the radial roll gap between the mandrel and forming roll is closed over successive rotations to reduce the radial wall thickness;

- the axial rolls are also driven and their roll gap can be closed to reduce the ring's axial height;

- the guide rolls apply a restoring moment to prevent the centre of the ring drifting away from the $X$-axis;

- the ring diameter grows as the cross section area reduces over successive radial and axial passes, up to reaching the target diameter.

Conventional industrial ring rolling processes have closed-loop control systems for the various degrees of freedom of the different rolls [13]. Usually, a single-point measurement of the ring diameter is taken using a contacting 

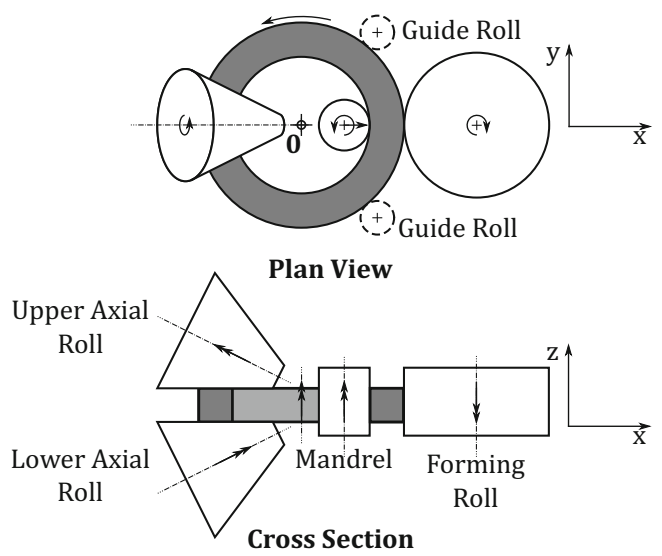

Fig. 1 Configuration of a conventional RARR machine

roll or a laser distance sensor. The displacements to be prescribed for the different rolls are determined based on this measurement and a rolling curve, which prescribes the path from initial to final cross section [14]. Effectively, the closed-loop control system only accounts for the ring diameter, whereas the evolution of the cross-section is controlled with and open-loop tool path.

The use of a camera measurement system in this work enables the control of ring curvature and radial thickness along its circumference. Recent developments in sensing in ring rolling using photogrammetry have shown that it is a practical observation technique $[4,6,15,16]$. [6] showed that the function of the guide rolls could be replaced by controlling the relative driving speeds of the axial and forming rolls to keep the ring centred.

The majority of previous research in RARR has focused on improving the reliability of the conventional process or on the development of new process designs that increase the range of allowable ring geometry $[2,3]$. This relates mainly to non-rectangular ring cross sections, for which each desired cross section requires its own specific tool geometry $[10,20]$. Recently, a ring rolling process has been developed to produce rings with different cross sections, without using part-specific tooling $[8,9]$. Such an increase in production flexibility significantly extends the applicability of ring rolling for products, which are produced in small batches. The current work aims at extending the flexibility of ring rolling processes, through simultaneous control of variable radial thickness [6] and variable curvature [5]. It will be shown that creating rings with both variable radial thickness and variable curvature is possible using conventional rolling hardware with additional sensing and control.

\section{Geometry measurement during RARR}

Ideally, a ring will not deviate from a perfect cylindrical tube at any point during conventional RARR. However, in

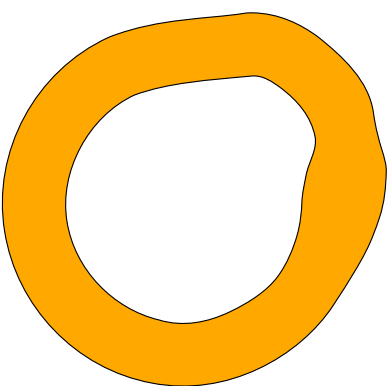

Fig. 2 An exemplar ring shape showing bends and variations in thickness. This is the idealised view of the upper surface

practice this is not always the case, as a forged preform has a rounded wall shape [21], and rolling errors, such as cavity formation, fishtail defects, non-circularity, conicity, dishing and waviness lead to deviations from a perfect cylindrical shape [2]. The use of single-point measurement devices, such as laser distance sensors, is sufficient for tracking the shape of a perfect cylindrical ring, but more advanced measurement systems must be used when the ring shape significantly deviates from the ideal shape. In this work, the rings are targeted to have variable thickness and curvature, leading to strong deviations from perfect circularity in the $X Y$-plane (Fig. 1). It is assumed that axial shape deviations (in $Z$-direction) will be small. Therefore the shape of the ring can be described by its intersection with the $X Y$-plane and its mean axial height. An exemplar ring shape with bending and significant variations in radial thickness is shown in Fig. 2. To address this measurement challenge, photogrammetry has been employed, which captures the two-dimensional shape of the top surface (where not occluded by tools). Ring rolling trials with clay material have been performed in this work, and the experimental setup including measurement equipment is shown in Fig. 3.

In addition to measuring the overall shape of the ring, it is also necessary to track the movement of the ring material relative to the tools. The relative speed of the forming roll

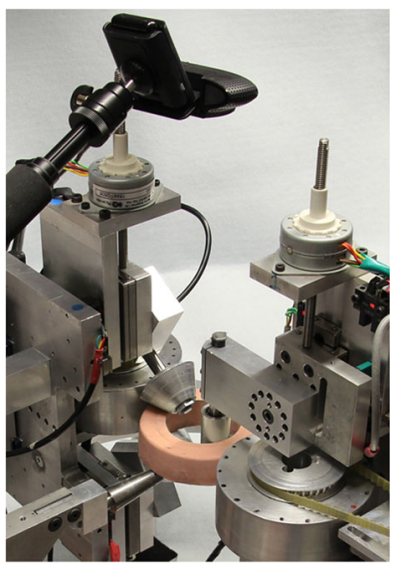

Fig. 3 Experimental setup for clay rolling trials, including video camera for recording the top surface of the ring [6] 
and the ring material are difficult to determine accurately due to changing contact conditions that lead to variable amounts of slipping. Therefore, an optical tracking strategy has been adopted. Surface features are invariably apparent in hot ring rolling, but for faster detection and more robust tracking, markers were applied to the rings in this work to locate the ring material. In practice, it is anticipated that periodic water jet spraying or high-temperature paint may be applied in the industrial process to assist with tracking. It may be possible to use digital image correlation to track arbitrary random surface features, but the computational effort in identifying known patterns is less than tracking random surface features, although this may be necessary when marking is not appropriate.

In this paper, the image processing procedure for tracking of the state of the ring is explained in Chapter "Image processing". Process control for variable thickness and variable curvature is discussed in Chapter "Process control". Chapter "Test results" presents trials for two different rings which have been simulated and produced with clay material. Finally, conclusions and discussion are found in Chapter "Conclusions and future research opportunities".

\section{Image processing}

The objective of the image processing algorithms is to estimate the current state of the ring geometry necessary for control of the process. A frame of video footage of the top surface of the ring (Fig. 4) is processed by a bespoke image-processing algorithm, which makes use of the nature of the ring shape to improve the speed, efficiency and accuracy of the numerical procedures. The estimated state of the ring's shape is then passed to the controller, which calculates the actuation required to forge the ring into the targeted shape. This section covers all parts of the image processing procedure, which are extracting ring boundaries ("Extracting ring boundaries"), local midline and thickness detection ("Ring midline and thickness"), tracking of ring rotation ("Ring rotation"), ring tracking in occluded regions ("Occluded regions and ring segmentation") and temporal filtering ("Temporal filtering").

\section{Extracting ring boundaries}

The boundary points of the ring in the RGB (red-greenblue) colour image are determined to nearest-pixel precision by segmenting the image into ring material and nonring material. The scene containing the ring, as shown in Fig. 4, is configured to make the segmentation and edge detection task simpler. The background is kept dark and non-reflective. In hot RARR, the ring glows with heat, which contrasts clearly with the dark background, but in

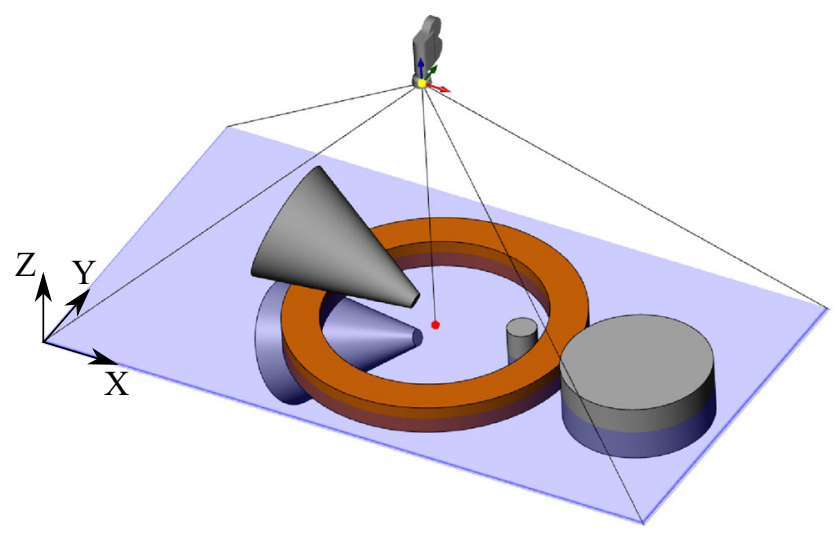

a

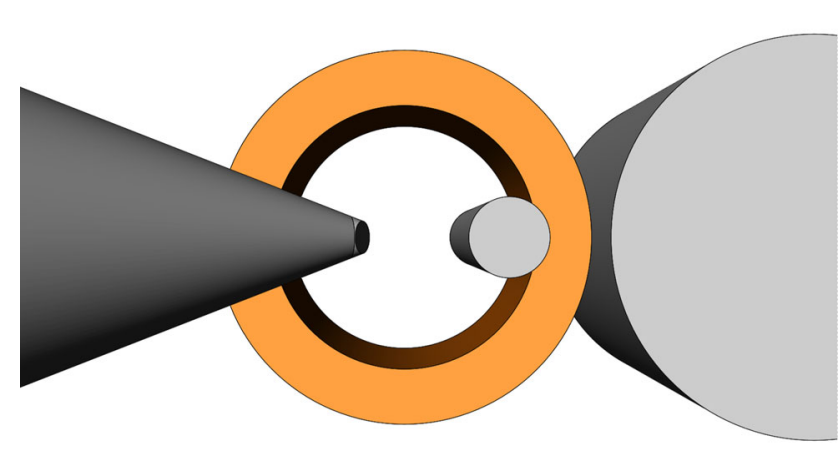

b

Fig. 4 Image (a) shows a 3D rendering of the important elements of the RARR machine in an idealised lighting scenario. Also shown is a camera with a wide field of view $\left(64^{\circ}\right)$. Image (b) shows the image that would be acquired by the camera. The top surface of the ring is occluded by the axial rolls and the mandrel. The inner cylindrical surface of the ring is lit by ambient light only

cold rolling the ring has to be illuminated by a light near the camera, so that the top surface of the ring is more strongly lit than the inner ring wall (the effect of which is shown in the simulated rendering of Fig. 4). The inner wall can also be painted black to enhance the contrast.

The full ring boundary detection process is outlined in Fig. 5. The segmentation is performed by creating a single user-defined combination function for the RGB colour channels so that the brightness of the ring material is maximised in the resultant grayscale image (ratio $100 \%$ for red, $51 \%$ for green and $2 \%$ for blue). The grayscale image is divided into ring and non-ring areas by a simple brightness threshold. The resulting image is filtered to select only the region with the largest contiguous area, which is the ring itself. The internal radial marker regions are removed by filling in any holes completely internal to the large area. The boundary pixels of the remaining regions are taken to be the edges of the visible ring material. Boundary positions close to the axial rolls and the radial roll gap are excluded because they do not represent the true boundary of the material. 

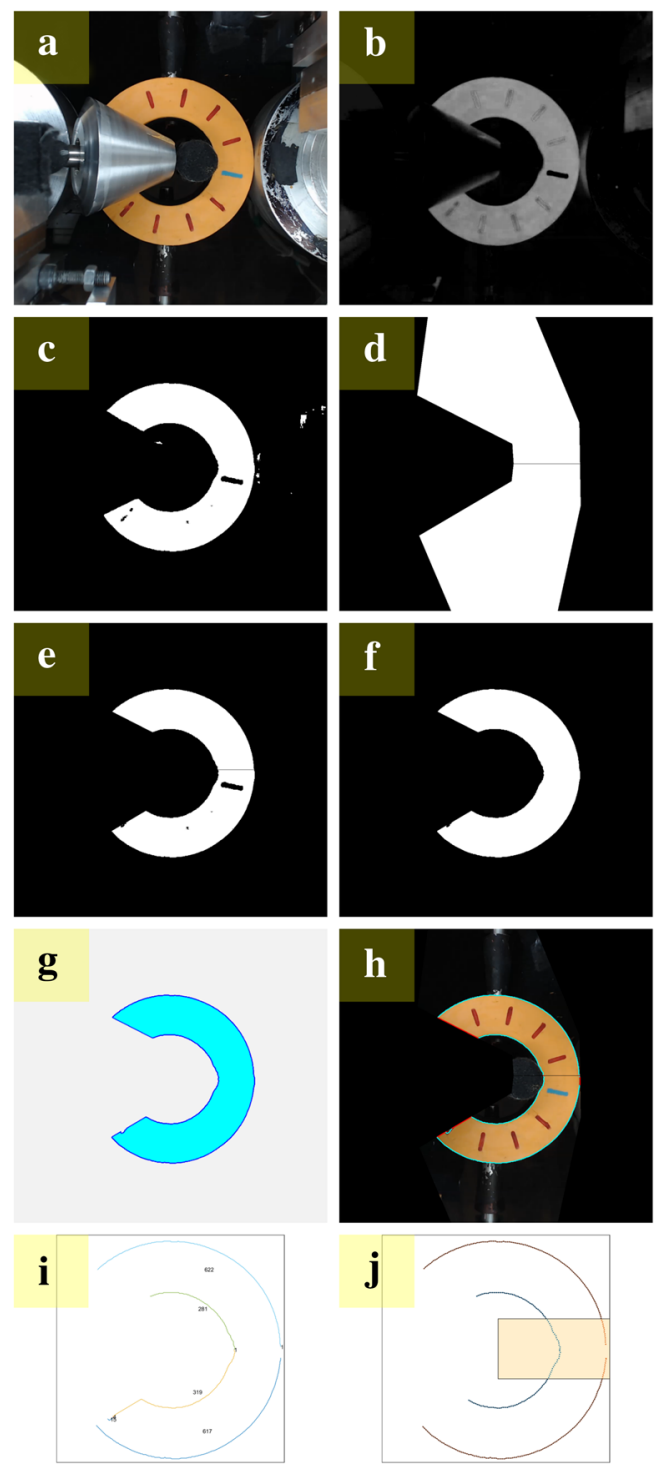

Fig. 5 Example of edge extraction for image of a ring inside the clay rolling machine. (A) original image; (B) grayscale image; (C) thresholded black and white (BW) image; (D) regions where the ring is not occluded; (E) BW image after ignoring occluded parts; (F) ring after dilation and erosion; (G) boundary edges of the segmented ring; (H) boundary edges on the original colour image; (I) continuous edge curve groups with their counts; $(\mathbf{J})$ remaining edge points and the radial forming region where edge points are also removed

Additional filtering of the ring edges is applied by interpolating the radii (calculated relative to a centre determined by fitting an ellipse to the boundary) of the boundaries linearly with angle. This reduces the number of erroneous boundary points because the paths of the boundaries are close to tangential.

\section{Ring midline and thickness}

The objective of the image processing algorithms is to estimate the current state of the ring geometry necessary

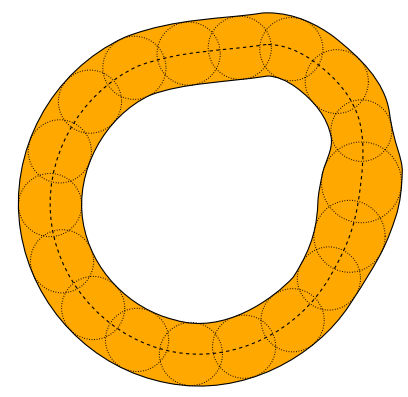

Fig. 6 The ring midline is calculated as the curve that connects the centres of the largest circles that fit touching, and tangential to, the inner and outer edges of the ring

for control of the process. The ring is assumed to maintain a rectangular wall cross-section, and therefore the state of geometry can be described by the ring's axial height and radial thickness along a closed path within the ring wall. It is assumed that the ring height is constant across the ring, and that the derivative of the path is continuous along the closed path - the path stretches and bends only.

As plastic deformation is a local state variable defined at each material point, it is essential to prescribe and track the evolution of plastic deformation relative to each material point. Therefore, the prescribed thickness and curvature of the ring is defined relative to each slice of ring material. Since the total ring volume remains constant in RARR, the ring material is parametrized as the fraction of total ring volume, $v_{f}(s)=v(s) / V_{T}$, along the path $s$. This Lagrangian approach to tracking is essential for comparison between the current state of geometry and the targeted curvature and thickness evolution.

The ideal closed tangential path along the ring's wall is the curve that, with height and radial thickness defined perpendicular to the path, will result in the correct $v_{f}$ at any location along the path. This curve is the 'mean' curve through the ring wall, which we will denote as the 'midline'. For most shapes encountered during ring-rolling the midline is closely related to the longest 'medial-axis' or the 'skeleton' of the ring's two-dimensional shape in plane-view. Figure 6 shows the graphical construction for estimating an ideal midline from the boundaries of the upper surface of an arbitrary ring.

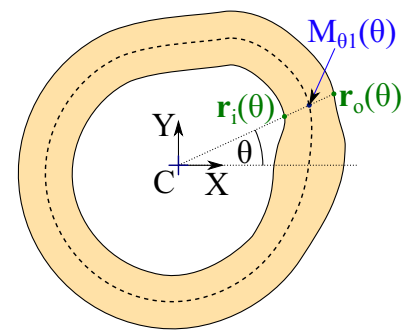

Fig. 7 The approximate midline is calculated from the mean of the radial intersections with the ring boundaries 
In practice, this construction of the midline is very time consuming. A faster estimate of the midline (Fig. 7) is produced by fitting an ellipse to all edge points to determine the ring's centre $\mathbf{C}$ and the inner and outer radii, $\mathbf{r}_{i}(\theta)$ and $\mathbf{r}_{o}(\theta)$, and then calculating their mean at all discernible angles to estimate the coordinates of the midline as function of the angle:

$\mathbf{M}_{\theta 1}(\theta)=\left(\mathbf{r}_{o}(\theta)+\mathbf{r}_{i}(\theta)\right) / 2$,

The initial midline estimate is improved with the following procedure (Fig. 8). First, the normal to $\mathbf{M}_{\theta 1}(\theta)$ and the intersections along this normal with the inner and outer edge boundaries are calculated. The distance between the intersection points at each angle, and their mean position, provide a reasonably accurate estimate of the normal thickness, $T_{\theta}(\theta)$ and the midline curve, $\mathbf{M}_{\theta}(\theta)=$ $[x(\theta), y(\theta)]^{T}$ respectively. To calculate the thickness with volume fraction $v_{f}$, the arc length around the midline is calculated, which produces $\mathbf{M}_{s}(s)$ and $T_{S}(s)$. Given the assumption of constant height $h$ along the ring, the volume fraction along the path is found with:

$v_{f}(s)=\frac{1}{V_{T}} h \int_{0}^{s} T_{s}(\tau) d \tau$

\section{Ring rotation}

To control the deformation of the ring material, it is necessary to know $v_{f}(s)$ along the midline to align the targeted thickness and curvature relative to the current state. The speed of the ring cannot be accurately estimated by the speed of the rollers because they slip against the surface of the ring, so surface marking was employed which could be measured from the same images used to estimate the ring's boundary shape.

To estimate the position of the 'origin' in the ring, and $v_{f}$ from there, the tracking of only one location on the ring is required, and the position of the remainder of the midline can be estimated by integrating height and thickness along its length. However, this is not accurate when assumptions about the shape have been violated or disturbances in sensor data are present. Also, in practice, large fractions of the ring

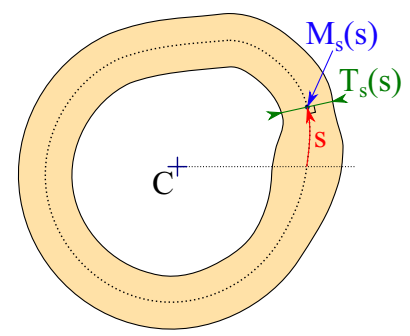

Fig. 8 The midline is parametrised by arc length and the thickness estimate is found using the distance between the normal intersections with the boundaries are periodically occluded, so the origin point would be lost. The practical solution to these issues in this work has been to use 12 initially-evenly-spaced markers and then estimate $v_{f}(s)$ in between, only over $1 / 12$ th of the ring. It is possible to increase the number of markers that are tracked, but more markers could be easily confused from one frame to the next, if they are closely spaced. Fewer markers could also be applied, but the error in estimating $v_{f}$ over a larger midline section would increase, and when fewer markers are visible between the tools there is a greater chance of losing the estimate of the origin position entirely if other disturbances prevent the tracking of some markers.

The markers take the form of radial lines of colour that contrast with the ring material, as shown in Fig. 9. The intersection between the midline and the radial line is the position in the material that is tracked. The markers are radial (as opposed to, say, dots) so that when the ring material is deformed and the markers deform by shearing and stretching, their intersection with the midline remains approximately constant and visible until the end of forming. The 'origin' of the ring is defined as the $s=0$ position (2) and has a different colour than the other 11 markers.

\section{Occluded regions and ring segmentation}

When the ring passes under the axial rolls or through the radial roll gap, its upper surface edges are occluded from view by the camera. In these zones the thickness of the ring material perpendicular to the midline and the length of the midline is assumed not to change until it crosses the $X Z$ plane of the mill (Fig. 1). The thickness of the ring at a particular volume fraction along the midline changes as it passes through the rollers, and it is difficult to accurately predict the change in thickness without full knowledge of the parameters governing the rolling conditions, such as friction, aspect ratio, temperature, etc. For this reason, the ring thickness and midline length between the roll gap exit side of the $X Z$-plane and the point at which the ring

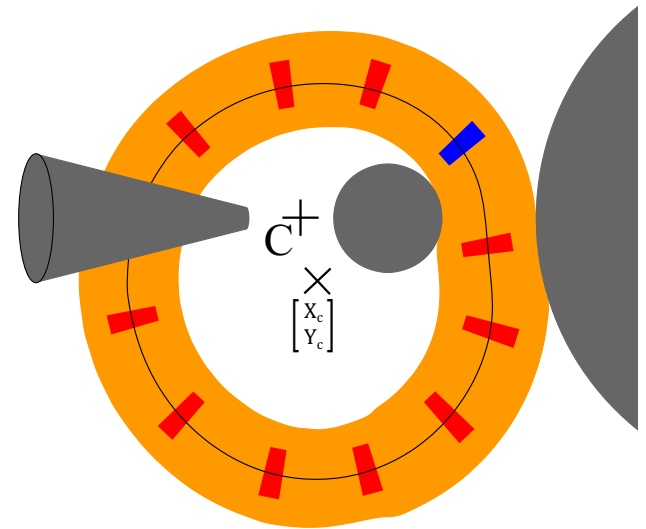

Fig.9 A marked arbitrary ring shape with the midline, machine centre and ring centre identified 
reappears in view of the camera are estimated by assuming that the thickness of the ring is equal to the roll gap as it passed through. The midline length is calculated by knowing the volume of this region and assuming uniform ring height in this region. Practically, the region after the roll gap does not need to be estimated because it will be measured properly when the camera can see it again, and only the estimated geometry of the material that is about to enter the roll gap is needed for the controller.

It is important to know the volume fraction of the ring that is about to cross the $X Z$-plane in order to calculate the actuation needed. When implementing the controller, the finite time taken to process the sensor data requires the controller to estimate the control actuation for a whole segment of the ring about to enter the roll gap. In subsequent sections a 'segment' of the ring will be referred to, which is, ideally, a small volume fraction $(<1 \%)$ of the ring and is assumed to be cuboid, with its length along the midline much shorter than the perpendicular directions. Figure 10 shows a ring split into 96 segments to visualise this scheme.

\section{Temporal filtering}

Once the material is tracked, the thickness at any particular volume fraction is measured repeatedly (where visible) and the thickness should remain constant at a given volume fraction $v_{f}$ between the exit side of one roll gap until entering the following roll gap. This permits the normal thickness estimates at all unnoccluded positions to be improved by filtering their thicknesses over several frames using median and moving-average filters. This filtering helps alleviate any errors in edge detection due to image noise, lighting changes, etc.

\section{Process control}

The photogrammetric procedure outlined in the previous section enables continuous tracking of the midline $\mathbf{M}_{s}(s)$, thickness $T_{s}(s)$ and volume fraction $v_{f}(s)$ as function of the path length $s$ (with $s=0$ being the 'origin' of the ring). These continuous measurements are used for variable

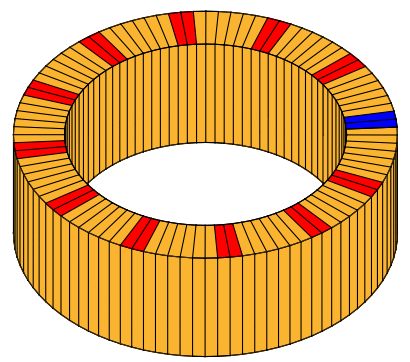

Fig. 10 The ring divided into segments thickness and curvature control. The radial gap between the mandrel and the forming roll is used to control the ring thickness, whereas the axial roll speed is used to control the local ring curvature. Hence, control of local thickness and local curvature are decoupled. In this chapter, the mechanisms for control of ring thickness and curvature are discussed. Open-loop scheduling and modelling for feedback control of ring thickness are discussed in "Open-loop radial gap planning for thickness control" and "Modelling for thickness feedback control" respectively. Open-loop scheduling and modelling for feedback control of ring curvature are discussed in "Open-loop curvature planning" and "Modelling for curvature feedback control" respectively.

\section{Open-loop radial gap planning for thickness control}

Due to the mechanical constraints of the rolling process, the thickness reduction per revolution must be limited. Conventional rolling closes the radial roll gap at a constant rate until the ring has reached its target diameter (wherupon the radial roll gap is held constant), whilst the forming roll rotates at a constant rate, which leads to a reduction in the ring's angular velocity as its radius grows.

With a variable wall thickness target, the radial roll gap must change much more rapidly in the final revolution of the ring to maintain contact with the ring. However, there is some choice in specifying the revolution in which the plastic change in thickness is obtained. For instance the ring could be rolled in the conventional manner, keeping constant thickness throughout the ring, until the thickness is at the maximum targeted thickness in the reference ring, and only then start reducing the thickness of the thinner segments in subsequent passes. In this work, the plastic change in thickness is divided into equal fractions per revolution to achieve the targeted thickness in a defined number of revolutions, but without exceeding the maximum thickness reduction permitted by the mechanics of the rolling conditions. The open-loop tool path is determined under the assumption of rigid plastic material behaviour, meaning that the resulting ring thickness is equal to the roll gap. In "Modelling for thickness feedback control" , a process model for feedback control is developed. This model accounts for springback, machine compliance and transverse strain in the axial roll pass. It will be shown that this model can be used to determine an improved open-loop tool path.

\section{Modelling for thickness feedback control}

The open loop tool path is determined under the assumption of rigid plastic material behaviour. However, axial passes increase the radial thickness due to the Poisson effect, 
and the thinning effect of radial passes is reduced by machine compliance and material springback (part of the deformation in the forming zone is elastic, and these compressive elastic strains reduce after leaving the forming area, leading to less thinning). Therefore, feedback control may be employed to increase forming accuracy. In this section, a feedback control algorithm is proposed based on mechanical modelling of the ring rolling process.

It is assumed that the cross section of each ring segment remains rectangular during rolling, and can be described with an axial thickness $t^{a}$ and a radial thickness $t^{r}$. In this work, variable radial thickness is targeted, while the axial roll gap is kept constant in order to keep constant axial thickness during rolling. Let the radial thickness of a segment before the $k$ th rotation be $t_{k}^{r}$, after the $k$ th radial rolling pass be $t_{k+0.5}^{r}$ and after the $k$ th rotation through both rolling stages be $t_{k+1}^{r}$. The roll gap between the mandrel and forming roll for that segment in the $k$ th rotation is $u_{k}^{r}$.

In the following derivation, it is assumed that the thickness evolution at a specific segment is independent of the thickness evolution in neighbouring segments. The derivation holds for a single ring segment, and is decoupled from other ring segments. It is key to keep in mind that the time index $k$ represents different passes through the roll gaps of a single ring segment only. One could extend the model in order to relate the thickness evolution of neighbouring segments, but that it not considered in this work.

Due to springback and machine compliance, the radial thickness immediately after the radial stage is larger than the mandrel-forming-roll gap. It is assumed that the thickness after rolling $t_{k+0.5}^{r}$ is independent of the incoming thickness $t_{k}^{r}$, and can be modelled as:

$t_{k+0.5}^{r}=c_{r} u_{k}^{r}$

where $c_{r}$ is a constant dependent on the rolling conditions. As the thickness after the pass will not be smaller than the roll gap, the condition $c_{r} \geq 1$ holds. The thickness after the pass depends on the machine compliance and on the amount of springback. It is expected to be in the range of $1 \leq c_{r} \leq 1.1$ for rolled metals.

Assuming that the resultant change of geometry caused by a rolling stage has no shear component and is uniform across the segment (so that cuboids remain cuboid), then the average plastic strains in the non-principal directions for the whole segment can be determined from the plastic ratio, $\lambda$, of the current stress state determined by the rolling conditions. In the radial rolling stage (where the majority of the strain achieved is radial) the plastic axial strain, $\epsilon_{r}^{a}$, is then given by:

$\epsilon_{r}^{a}=-\lambda_{r} \epsilon_{r}^{r}$ where $\epsilon_{r}^{r}$ is the plastic radial strain. Similarly the plastic radial strain after the axial rolling stage:

$\epsilon_{a}^{r}=-\lambda_{a} \epsilon_{a}^{a}$

where $\epsilon_{a}^{a}$ is the axial strain after the axial stage. For example, neglecting friction and without compressive stresses in the tangential ring direction, the stress state would be uniaxial, with a plastic ratio of $\lambda_{r}=0.5$. Due to compressive stresses in tangential ring direction [12], the plastic ratio will be slightly larger than 0.5 .

From the definition of true strain given by $\epsilon=\ln \left(L / L_{0}\right)$, where $L_{0}$ is the original length and $L$ is the resultant length, the plastic radial strain for the segment after the radial stage only is thus given by:

$\epsilon_{r}^{r}=\ln \left(\frac{t_{k+0.5}^{r}}{t_{k}^{r}}\right)$

giving the axial strain after radial rolling:

$\epsilon_{r}^{a}=-\lambda_{r} \epsilon_{r}^{r}=\ln \left(\left(\frac{t_{k+0.5}^{r}}{t_{k}^{r}}\right)^{-\lambda_{r}}\right)$

As the axial thickness is targeted to remain constant during rolling, it is assumed that the axial roll gap is set such that the axial thickness after each axial pass is equal to the targeted axial thickness, $t_{k}^{a}=t_{k+1}^{a}=\bar{t}^{a}$. The axial height after the radial stage, $t_{k+0.5}^{a}$, can be taken from $\epsilon_{r}^{a}=$ $\ln \left(t_{k+0.5}^{a} / t_{k}^{a}\right)$ :

$t_{k+0.5}^{a}=\left(\frac{t_{r}^{k+0.5}}{t_{k}^{r}}\right)^{-\lambda_{r}} \bar{t}^{a}$

In the axial rolling stage, the segment is compressed axially under different rolling conditions, and the ratio of strains is modelled using $\lambda_{a}$. The axial strain after the axial stage is given by $\epsilon_{a}^{a}=\ln \left(t_{k+1}^{a} / t_{k+0.5}^{a}\right)$, and the final axial height after the axial stage is again $\bar{t}^{a}$. The radial strain caused by the axial rolling stage can now be computed using Eq. 5:

$\epsilon_{a}^{r}=\ln \left(\frac{t_{k+1}^{r}}{t_{k+0.5}^{r}}\right)=\ln \left(\left(\frac{\bar{t}^{a}}{t_{k+0.5}^{a}}\right)^{-\lambda_{a}}\right)$

and then the final radial size can be obtained:

$$
\begin{aligned}
& t_{k+1}^{r}=\left(\frac{\bar{t}^{a}}{t_{k+0.5}^{a}}\right)^{-\lambda_{a}} t_{k+0.5}^{r} \\
& t_{k+1}^{r}=\left(\left(\frac{t_{k+0.5}^{r}}{t_{k}^{r}}\right)^{\lambda_{r}}\right)^{-\lambda_{a}} t_{k+0.5}^{r} \\
& t_{k+1}^{r}=\left(\frac{t_{k+0.5}^{r}}{t_{k}^{r}}\right)^{-\lambda_{r} \lambda_{a}} t_{k+0.5}^{r} \\
& t_{k+1}^{r}=t_{k}^{r\left(\lambda_{r} \lambda_{a}\right)} t_{k+0.5}^{r}{ }^{\left(1-\lambda_{r} \lambda_{a}\right)}
\end{aligned}
$$


Substituting $t_{k+0.5}^{r}$ from Eq. 3 gives $t_{k+1}^{r}$ in terms of $t_{k}^{r}$ and $u_{k}^{r}$ :

$t_{k+1}^{r}=\left(t_{k}^{r}\right)^{\lambda_{r} \lambda_{a}}\left(c_{r} u_{k}^{r}\right)^{\left(1-\lambda_{r} \lambda_{a}\right)}$

This system model can be normalised by dividing the new thickness $t_{k+1}^{r}$ and the roll gap $u_{k}^{r}$ by the input thickness $t_{k}^{r}$, turning Eq. 11 into:

$\hat{t}_{k+1}^{r}=\left(c_{r} \hat{u}_{k}^{r}\right)^{\left(1-\lambda_{r} \lambda_{a}\right)}$

where $\hat{t}_{k+1}^{r}=t_{k+1}^{r} / t_{k}^{r}$ and $\hat{u}_{k}^{r}=u_{k}^{r} / t_{k}^{r}$. As $c_{r}$ is just larger than 1 and $\hat{u}_{k}^{r}$ is just smaller than 1, Eq. 12 is linearised about $c_{r} \hat{u}_{k}^{r}=1$ :

$\hat{t}_{k+1}^{r} \approx c_{r}\left(1-\lambda_{r} \lambda_{a}\right) \hat{u}_{k}^{r}+\lambda_{r} \lambda_{a}$

The non-normalised system then becomes:

$t_{k+1}^{r} \approx c_{r}\left(1-\lambda_{r} \lambda_{a}\right) u_{k}^{r}+\lambda_{r} \lambda_{a} t_{k}^{r}$

The term $c_{r}$ relates to reduced thinning due to springback and machine compliance, and the term $\lambda_{r} \lambda_{a}$ relates to transverse strain in the radial and axial pass. Without reduced thinning $\left(c_{r}=1\right)$ and transverse strain $\left(\lambda_{r} \lambda_{a}=0\right)$, the equation reduces to the assumption used for determining the open loop path: $t_{k+1}^{r}=u_{k}^{r}$. In order to determine the control action, Eq. 14 may be inverted for $u_{k}^{r}$ :

$u_{k}^{r}=\left(c_{r}\left(1-\lambda_{r} \lambda_{a}\right)\right)^{-1} t_{k+1}^{r}-\lambda_{r} \lambda_{a}\left(c_{r}\left(1-\lambda_{r} \lambda_{a}\right)\right)^{-1} t_{k}^{r}$

Consider $\bar{t}_{k}^{r}$ and $\bar{t}_{k+1}^{r}$ to represent the open loop tool path. Equation 15 can now be rewritten to:

$$
\begin{aligned}
u_{k}^{r}= & \left(c_{r}\left(1-\lambda_{r} \lambda_{a}\right)\right)^{-1} \bar{t}_{k+1}^{r}-\lambda_{r} \lambda_{a}\left(c_{r}\left(1-\lambda_{r} \lambda_{a}\right)\right)^{-1} \bar{t}_{k}^{r} \\
& -\lambda_{r} \lambda_{a}\left(c_{r}\left(1-\lambda_{r} \lambda_{a}\right)\right)^{-1} e_{k}^{r}
\end{aligned}
$$

with $e_{k}^{r}=\left(t_{k}^{r}-\bar{t}_{k}^{r}\right)$. The first two terms form an adapted open loop tool path that takes into account the predicted effects of reduced thinning and transverse strain. The third term represents the feedback control action which reacts on the measured error $e_{k}^{r}$. The control action is proportional only. When a series of final passes is performed with constant reference thickness $\bar{t}_{\text {final }}^{r}$, the control action $u_{k}^{r}$ stabilizes to a constant value. Assuming that the developed model is correct but that the model parameters $\lambda_{a}, \lambda_{r}$ and $c_{r}$ are not exactly known, the final thickness $t_{\text {final }}^{r}$ can be determined by calculating the control action from Eq. 16 with the model parameters $\lambda_{a}^{\mathrm{M}}, \lambda_{r}^{\mathrm{M}}$ and $c_{r}^{\mathrm{M}}$, and inserting the control action in Eq. 14 using the real but unknown parameters $\lambda_{a}^{\mathrm{R}}, \lambda_{r}^{\mathrm{R}}$ and $c_{r}^{\mathrm{R}}$. Using the condition that the thickness stabilizes to $t_{k+1}^{r}=t_{k}^{r}$, it follows that the final thickness is:

$\frac{t_{\text {final }}^{r}}{\bar{t}_{\text {final }}^{r}}=\frac{c_{r}^{\mathrm{R}}}{c_{r}^{\mathrm{M}}\left(1-\lambda_{r}^{\mathrm{M}} \lambda_{a}^{\mathrm{M}}\right)+c_{r}^{\mathrm{R}} \lambda_{r}^{\mathrm{M}} \lambda_{a}^{\mathrm{M}}}$ which is only equal to 1 when $c_{r}^{\mathrm{R}}=c_{r}^{\mathrm{M}}$. In order to avoid a final shape error due to modelling errors, a PI controller can be used:

$u_{k}^{r \mathrm{FB}}=-K_{p} e_{k}^{r}-K_{i} \sum_{i=1}^{k} e_{i}^{r}$ form:

The control equation can be rewritten in an incremental

$u_{k}^{r \mathrm{FB}}=u_{k-1}^{r \mathrm{FB}}-K_{p}\left(e_{k}^{r}-e_{k-1}^{r}\right)-K_{i} e_{k}^{r}$

Finally, the controller equations are:

$$
\begin{aligned}
u_{k}^{r}= & u_{k}^{r \mathrm{FF}}+u_{k}^{r \mathrm{FB}} \\
u_{k}^{r}= & u_{k}^{r \mathrm{FF}}+u_{k-1}^{r \mathrm{FB}}-K_{p}\left(e_{k}^{r}-e_{k-1}^{r}\right)-K_{i} e_{k}^{r} \\
u_{k}^{r \mathrm{FF}}= & \left(c_{r}\left(1-\lambda_{r} \lambda_{a}\right)\right)^{-1} \bar{t}_{k+1}^{r} \\
& \quad-\lambda_{r} \lambda_{a}\left(c_{r}\left(1-\lambda_{r} \lambda_{a}\right)\right)^{-1} \bar{t}_{k}^{r}
\end{aligned}
$$

The control system is schematically shown in Fig. 11 . The gain factors $K_{p}$ and $K_{i}$ are chosen relative to the gain factor determined in Eq. 16:

$$
\begin{aligned}
K_{p} & =\alpha \lambda_{r} \lambda_{a}\left(c_{r}\left(1-\lambda_{r} \lambda_{a}\right)\right)^{-1} \\
K_{i} & =\beta \lambda_{r} \lambda_{a}\left(c_{r}\left(1-\lambda_{r} \lambda_{a}\right)\right)^{-1}
\end{aligned}
$$

Choosing $\alpha=1$ and $\beta=0$ corresponds with the control action given in Eq. 16. The actual values of $\alpha$ and $\beta$ may be tuned around 1. Under the assumption that the model is correct but that the model parameters are not known, the following stability criterion can be derived by determining the set of model parameters $c_{r}^{\mathrm{M}}, \lambda_{a}^{\mathrm{M}}, \lambda_{r}^{\mathrm{M}}, \alpha$ and $\beta$ at the onset of system instability, causing the thickness to jump between two values at consecutive rotations $\left(\Delta t_{r}^{k+1}=-\Delta t_{r}^{k}\right)$ around the reference thickness $\left(t_{r}^{k}-\bar{t}_{k}^{r}=\Delta t_{r}^{k} / 2\right)$ :

$\frac{c_{r}^{\mathrm{M}}\left(1-\lambda_{r}^{\mathrm{M}} \lambda_{a}^{\mathrm{M}}\right)}{\lambda_{r}^{\mathrm{M}} \lambda_{a}^{\mathrm{M}}\left(\alpha+\frac{\beta}{2}\right)}>\frac{c_{r}^{\mathrm{R}}\left(1-\lambda_{r}^{\mathrm{R}} \lambda_{a}^{\mathrm{R}}\right)}{1+\lambda_{r}^{\mathrm{R}} \lambda_{a}^{\mathrm{R}}}$

For illustration, it is assumed that the model parameters are correctly chosen $\left(\lambda_{a}^{\mathrm{M}}=\lambda_{a}^{\mathrm{R}}, \lambda_{r}^{\mathrm{M}}=\lambda_{r}^{\mathrm{R}}\right.$ and $\left.c_{r}^{\mathrm{M}}=c_{r}^{\mathrm{R}}\right)$ and that the value of $\lambda_{a}^{\mathrm{R}}=\lambda_{r}^{\mathrm{R}}=0.6$. In this case, it follows that $\alpha+\beta / 2<3.78$ is the condition for system stability. Additional constraints should be taken into account to ensure that the tool and the ring do not lose contact.

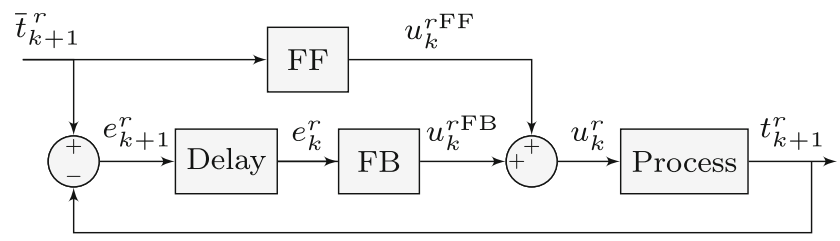

Fig. 11 Diagram for thickness control, with a feedback (FB) and feedforward (FF) loop 


\section{Open-loop curvature planning}

The curvature of the midline of a ring as a function of midline arc length, $\kappa^{s}(s)$, is calculated from the midline path using:

$$
\begin{aligned}
\mathbf{M}^{s}(s) & =[x(s), y(s)]^{T} \\
\kappa^{s}(s) & =\frac{x^{\prime}(s) y^{\prime \prime}(s)-y^{\prime}(s) x^{\prime \prime}(s)}{\left(\left(x^{\prime}(s)\right)^{2}+\left(y^{\prime}(s)\right)^{2}\right)^{3 / 2}} .
\end{aligned}
$$

$\kappa^{s}$ for a targeted ring shape is calculated in the same way from the targeted midline shape. When the targeted ring wall radial thickness is not constant, the curvature has to be calculated as a function of volume fraction around the ring to enable comparisons between current and targeted ring states, providing $\kappa^{v}\left(v_{f}\right)$. In addition, the curvature of the targeted ring shape is only achieved when the thickness has been reduced to the final targeted thickness and the total arc length, $S_{\max }$, has reached its targeted value too. To make comparisons of the state of curvature between the current and final shapes, the curvature can be normalised by multiplying by $S_{\max }$ to give a normalised curvature value:

$\hat{\kappa}^{v}\left(v_{f}\right)=S_{\max } \kappa^{v}\left(v_{f}\right)$

All circular rings have mean normalised curvature of $\hat{\bar{\kappa}}^{v}=2 \pi$ by definition.

An open-loop tool path can be constructed based on assumptions about the deformation mechanics. The change in curvature at the forming rolls depends on the rolling conditions and the constitutive behaviour of the ring material. If the material has rigid-plastic behaviour, there will be no springback and the state of a ring segment will not change after leaving the deformation zone. Hence, a first approximation for the rotation required is that the $X$-axis of the rolling mill should be held perpendicular to the targeted ring wall midline for the segment passing through the radial stage. For creating circular rings this means that the centre of the ring always lies on the $X$-axis. For desired ring shapes with midlines that differ from circular, for instance a smooth-cornered square as shown in Fig. 12, the open-loop control objective would move the ring relative to the mill as shown. The angle $\delta$ between the $X$-axis of the mill (the line of action of the radial rolling stage) and the line connecting the centre of the ring to the centre of the current segment passing through the radial roll gap can be calculated offline as a function of $v_{f}$.

The angle $\delta$ can be changed by adjusting the speed of the axial rolls relative to the forming roll so that the tangential ring speeds are not equal for both rolling stages. When the magnitude of the rolling speed at the axial roll gap is greater than at the radial roll gap, the axial rolls displace the ring wall in the $Y$-direction by a different amount to the radial

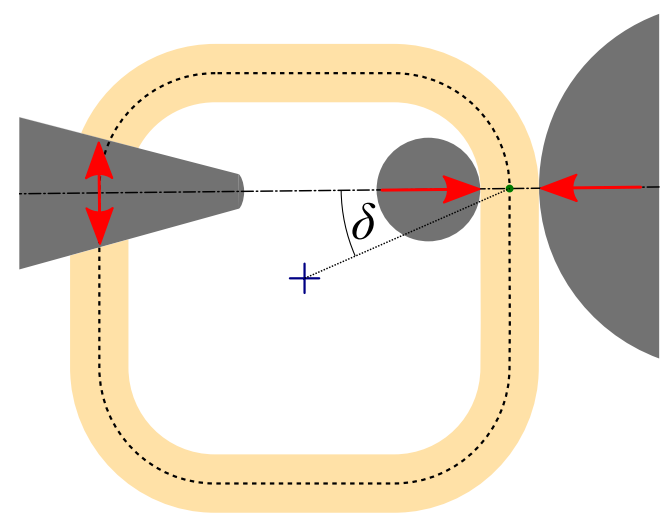

Fig. 12 The open-loop control objective for the example of a smoothcornered square ring

roll gap and this creates an 'opening' 1 or a 'closing' bending moment within the radial roll gap. If the bending moment exceeds the plastic bending moment for the ring wall within the radial roll gap then a plastic hinge forms there, causing a permanent change in ring wall curvature. Because the ring is a closed loop, there must be an opposite change in curvature in the ring wall remote from the bent region for compatibility to be maintained, but this is distributed over a longer arc length by elastic bending caused by the residual stresses. Assuming that the axial rolls impose a force on the ring which acts perpendicular to the mill axis (i.e. in $Y$ direction), the maximum bending moment in the ring occurs at the radial rolls. In combination with compressive forces applied by the mandrel-forming roll pair, the material will yield at the radial roll gap, leading to localisation of the plastic bending, which is useful for decoupling the effect of changing the curvature in one region of the ring wall from neighbouring regions.

The closed-loop positioning of the ring to achieve the predetermined $\delta$ angle is performed using an inner PID control loop for the speed of the axial rolls relative to the speed of the forming rolls about the nominal rolling velocity (Fig. 13).

\section{Modelling for curvature feedback control}

Simplifying assumptions about the mechanisms of curvature evolution in RARR have been used for construction of the open-loop curvature path in the previous section. In order to improve geometrical product accuracy, feedback control may be employed. A control diagram including open-loop and feedback control is shown in Fig. 13. Such a feedback control system can be tuned based on process

\footnotetext{
1'Opening' here means that the ring has reduced curvature and hence increased radius of curvature.
} 


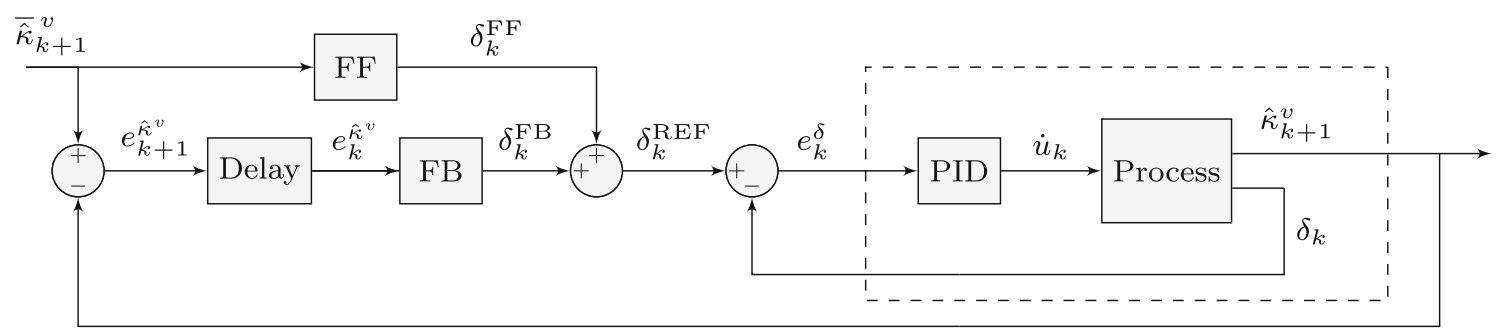

Fig. 13 Diagram for curvature control, with a feedback (FB) loop, a feedforward (FF) loop and an internal PID controller for control of the ring angle $\delta$

data or using a process model, similar to the analytic model used for thickness feedback control in "Modelling for thickness feedback control". Furthermore, a model may account for the change of process response with evolving product state, which will be difficult to identify based on process data only. In this section, it is discussed how such a model may be developed.

For construction of the open-loop tool path, it is assumed that no springback occurs and, therefore, that the midline must remain perpendicular to the $X$-axis of the rolling mill. Due to springback, additional curvature change must be generated by rotating the ring with an additional angle $\alpha$ (Fig. 14). Similarly to the model for local ring thickness control, a model that relates the angle $\alpha$ to the resulting curvature can be used to both improve the open-loop tool path as well as to estimate appropriate feedback control actions.

Developing a model for the curvature evolution in ring rolling is not as straight forward as for the thickness reduction. Several analytical models have been developed for ring rolling, with the objective to estimate ring growth rate (e.g [22]), process forces (e.g. [19]) or constraints of process conditions (e.g [7]). With respect to curvature evolution, [17] reviewed analytical and numerical curvature evolution models for the sheet rolling process, which has

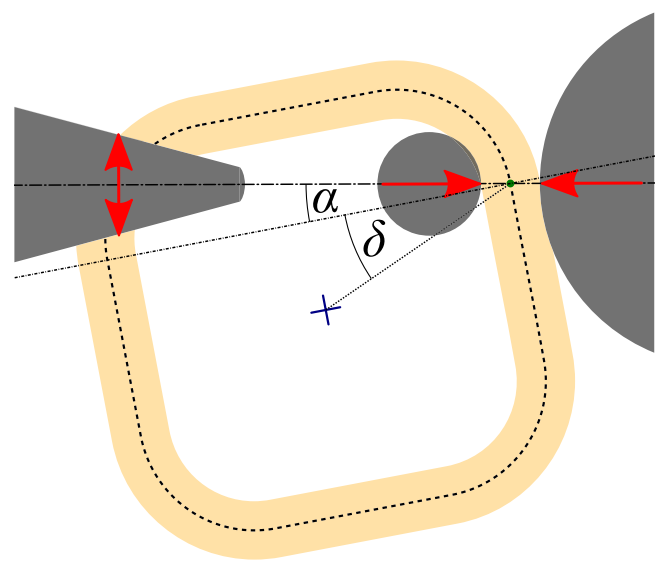

Fig. 14 Additional ring rotation $\alpha$ in order to account for springback strong similarities with the ring rolling process. They showed that these works present contradictory results, as curvature evolution depends on many factors and is therefore difficult to predict. Following to this work, [18] developed a modelling framework of the ring rolling process, which accounts for non-circular, non-centred and non-coaxial rings. He uses an extended slab method for the deformation zone and an elastic curved beam model for the rest of the ring. Although no appropriate coupling of these models was found to ensure system convergence, the equations can be used as a starting point for the development of a curvature evolution model for process control. As the extended slab model depends on the roll gap, the thickness reduction and curvature evolution models will become coupled, which must be accounted for in the controller design.

\section{Test results}

Past work has demonstrated that variable thickness rings with uniform curvature [4] and variable curvature rings with uniform thickness [5] can be created using conventional RARR hardware and the additional sensing described earlier. This paper has covered the theory behind the implementation of the control strategy in more depth for these two processes. We now present two trials of forming rings with combined varying thickness and varying curvature. The target geometries and open-loop tool paths are presented in "Target rings". The trials have been first simulated. The simulation procedure is explained in "Simulation procedure" and the results are presented in "Simulation results". Finally, experimental clay trial results are presented in "Clay trials".

\section{Target rings}

The two targeted shapes investigated here were: a D-ring with two short sections of thickened wall (Fig. 15a); and a smooth-cornered square ring with two opposite sides made thicker than the rest (Fig. 15b). These rings are both formed from rings with initial inner and outer diameters of $45 \mathrm{~mm}$ 


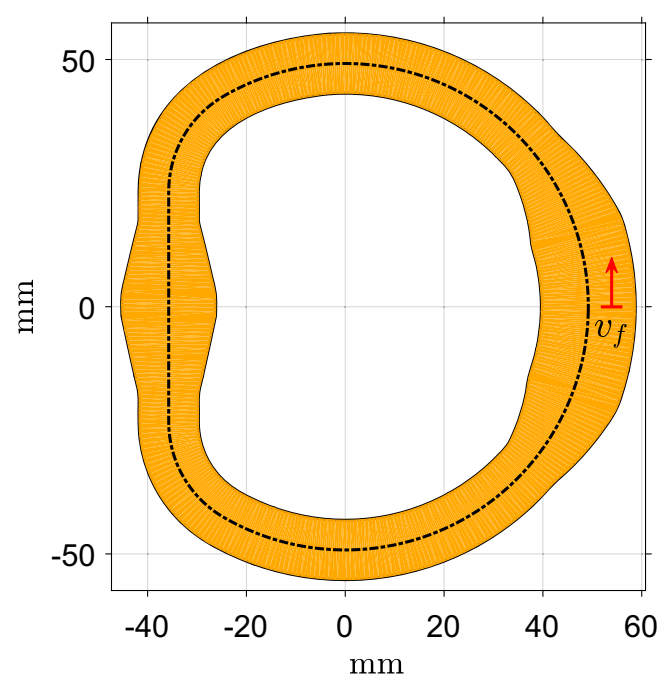

a

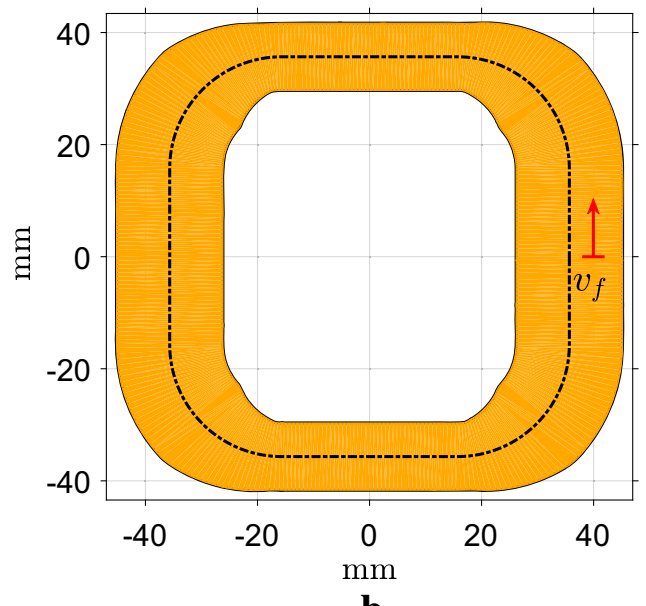

b

Fig. 15 a The D ring with two thick sections, one longer than the other, and both with smooth entry and exit gradients. b The Square ring with two opposite sides thicker.

and $85 \mathrm{~mm}$ respectively, and an axial height of $25 \mathrm{~mm}$, which is kept constant during rolling.

In Fig. 16, the curvature of the midline as a function of volume fraction can be seen for both shapes. In Fig. 17 the

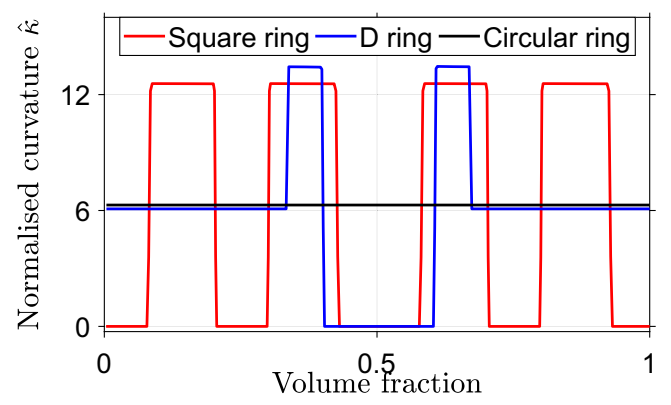

Fig. 16 Midline curvature targets for each shape

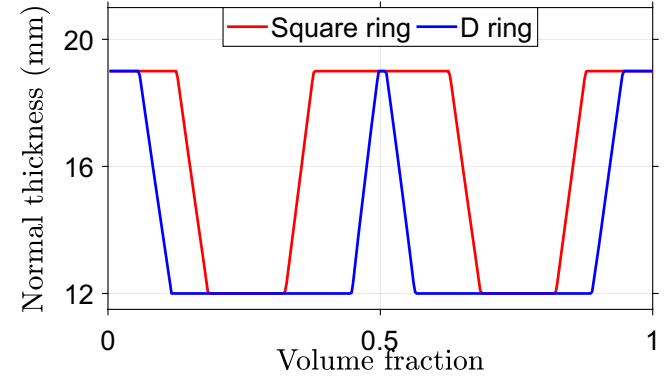

Fig. 17 Thickness targets for each shape

normal thickness as a function of volume fraction can be seen.

Figure 18 shows the evolution of the open-loop radial roll gap path with subsequent rotations of the ring for the Dring and Fig. 19 shows the same data for the square ring. In conventional ring rolling the radial roll gap decreases with constant gradient. In contrast, for the open-loop path roll gap here the total strain in each segment was divided into equal parts for the first 10 rotations and 5 subsequent rotations maintained the final thickness commanded of each segment. The used open-loop tool path does not account for the effect of transverse strain and reduced thinning, i.e. it is assumed that the radial thickness after both passes is equal to the roll gap $\left(t_{k+1}^{r}=u_{k}^{r}\right)$.

Figure 20 shows the growth of the total midline length planned by the open-loop roll-gap paths of Figs. 18 and 19 assuming that the axial height does not change. Figure 21 shows the final normal angle $\delta$, as function of volume fraction $v_{f}$ for both rings. The normal angle is used as the target for the internal PID controller of the speed of the axial rolls relative to the speed of the radial rolls.

\section{Simulation procedure}

The trials were conducted using the finite element method to simulate the mechanics of the rolling mill and the material deformation. The procedure was first outlined in [5] but can be summarised as follows: a 3D model of a ring is modelled using Abaqus Explicit with an additional user

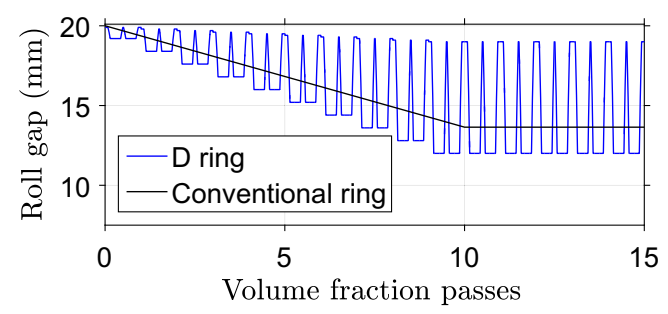

Fig. 18 The open-loop roll gap for the D ring and conventional ring 


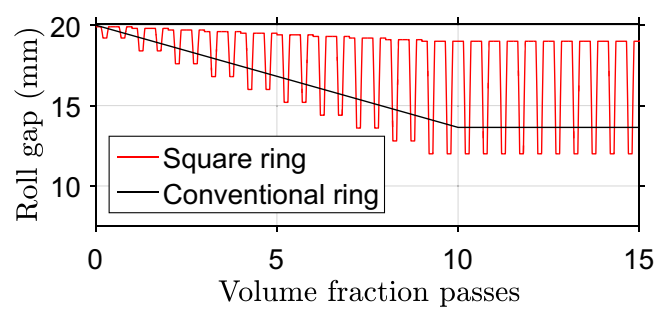

Fig. 19 The open-loop roll gap for the square ring and conventional ring

subroutine which periodically outputs the coordinates of the mesh to a text file. This text file was then read with Matlab and the ring was rendered as an image, as if being photographed by the optical camera in a real-world situation; the image processing and control algorithms were implemented in Matlab and a file containing actuation instructions was written and made available for Abaqus to read and implement over the course of the next period. In this way, the sensing and the control strategy did not have to be changed to control the simulated ring or a real-world rolling mill. The only significant operational difference between a real mill and the Abaqus-simulated mill is that the processing time could be, in effect, reduced to zero if so desired. However, a simulated delay in calculating the actuator settings was imposed to emulate the practical situation, although investment in improved computational hardware would reduce processing time for a real controller.

The simulations are performed to show that thickness and curvature of the rings can be controlled simultaneously. It is not intended to use the model for prediction of the manufacturing accuracy of the rolling procedure. Therefore, basic assumptions and settings have been used in the finite element model. A total of 1920 8-node linear brick elements with reduced integration have been used for the ring. A material model with a Young's modulus of $120 \mathrm{GPa}$, a Poisson's ratio of 0.3 , and a yield strength of $500 \mathrm{MPa}$ with linear work hardening is used.

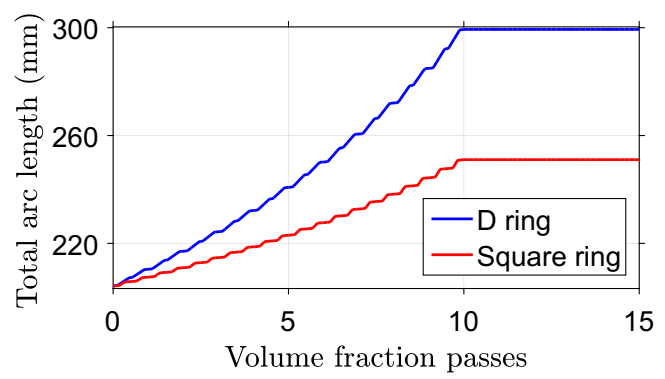

Fig. 20 This plot shows the total midline length of each ring, assuming the axial height remains constant

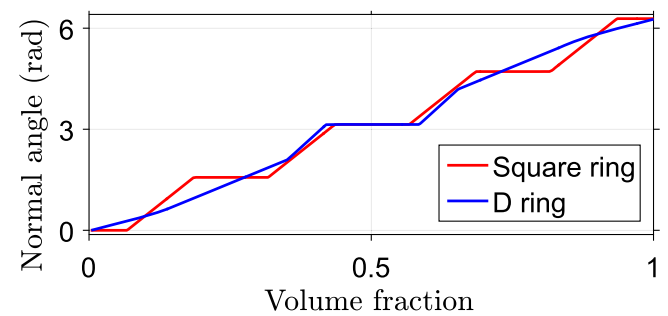

Fig. 21 Normal angle $\delta$ as function of volume fraction for both rings

\section{Simulation results}

Figures 22 and 23 show the final shapes obtained in the two simulations. The final midline total arc length of the D ring was $294.2 \mathrm{~mm}$, whereas the targeted value was $299.3 \mathrm{~mm}$. Similarly for the square ring the final midline total arc length was $252.5 \mathrm{~mm}$, whereas the target value was $251.0 \mathrm{~mm}$. The differences in the final dimensions can be attributed primarily to incorrect assumptions about the amount of elastic strain in the material.

Figures 24 and 25 show the curvature achieved in the final ring shapes. Figures 26 and 27 show the normal thickness achieved in the final ring shapes. It can be seen that the shapes are reasonably close to the reference shapes. Therefore, it can be concluded that the proposed sensing and control strategy can be used to produce rings with variable thickness and curvature. However, the observed deviations are too large for industrial application. Implementation of improved open-loop tool paths and feedback control for both thickness and curvature control as discussed in "Process control" can be used to improve production accuracy. It is however not in the scope of this work to investigate the effects of different control strategies on the accuracy of the rings.

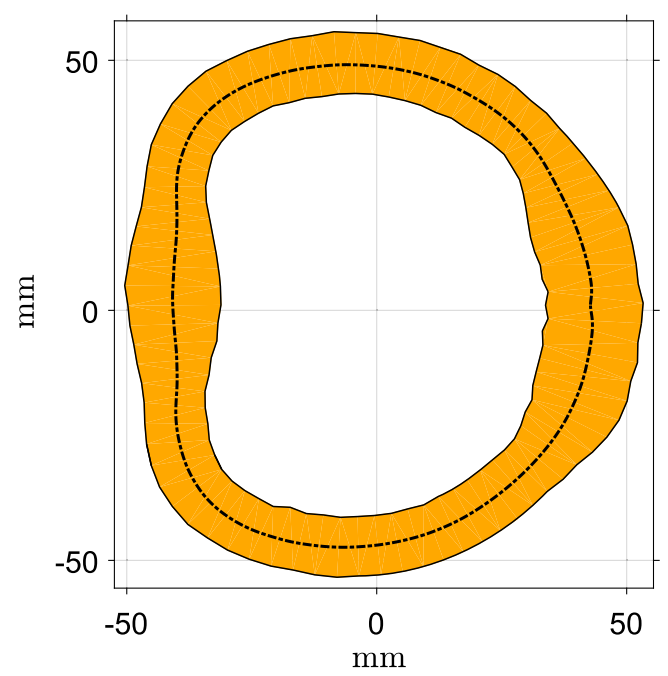

Fig. 22 The D ring ring final shape and its computed midline 


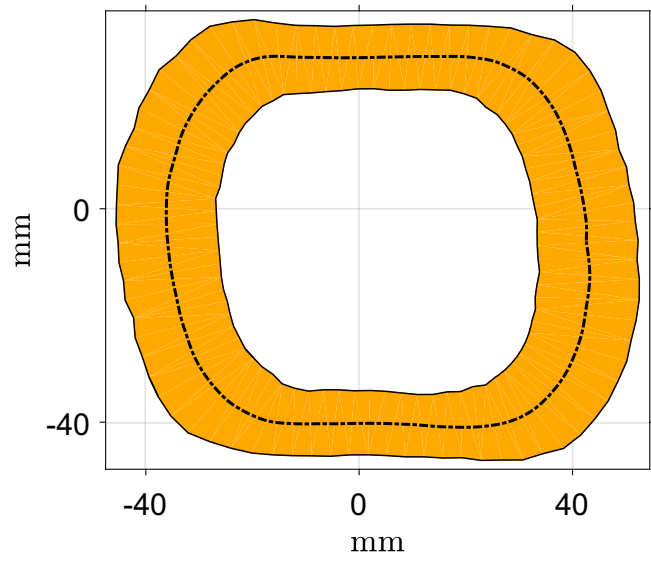

Fig. 23 The Square ring final shape and its computed midline

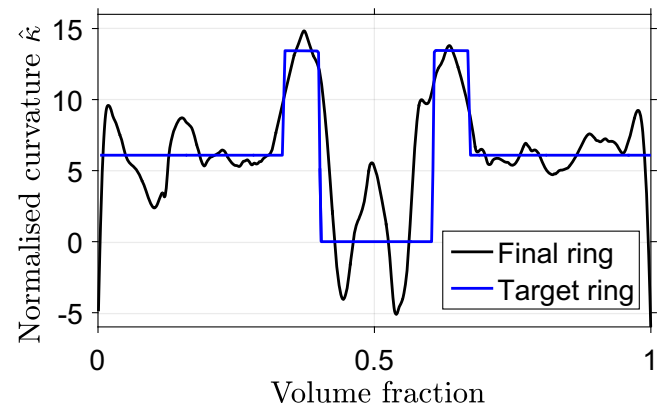

Fig. 24 The D ring ring midline curvature compared to the reference shape

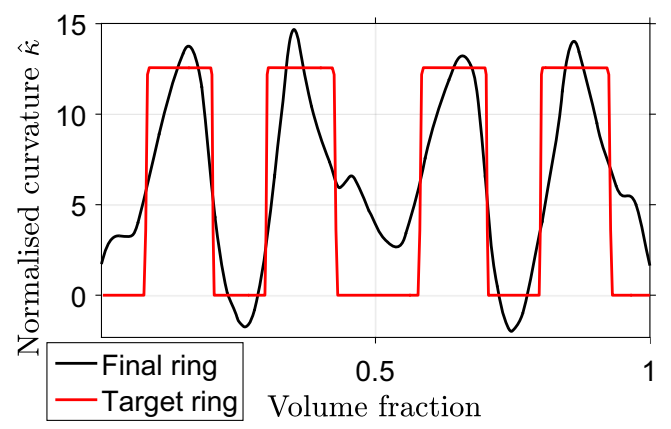

Fig. 25 The Square ring midline curvature compared to the reference shape

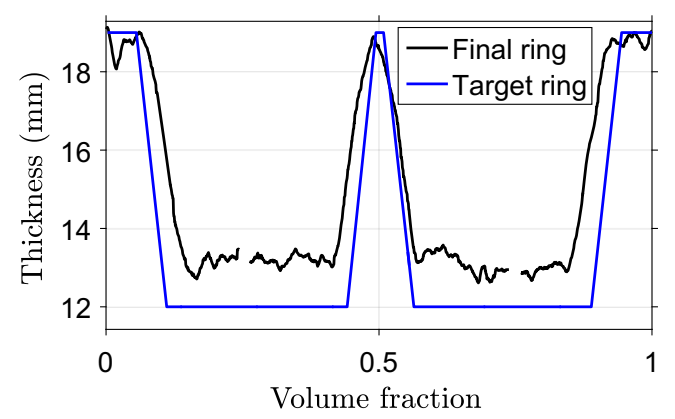

Fig. 26 The D ring ring normal thickness compared to the reference shape

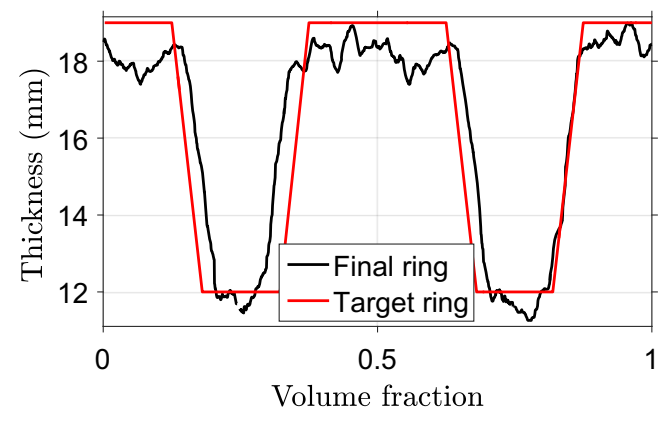

Fig. 27 The Square ring normal thickness compared to the reference shape

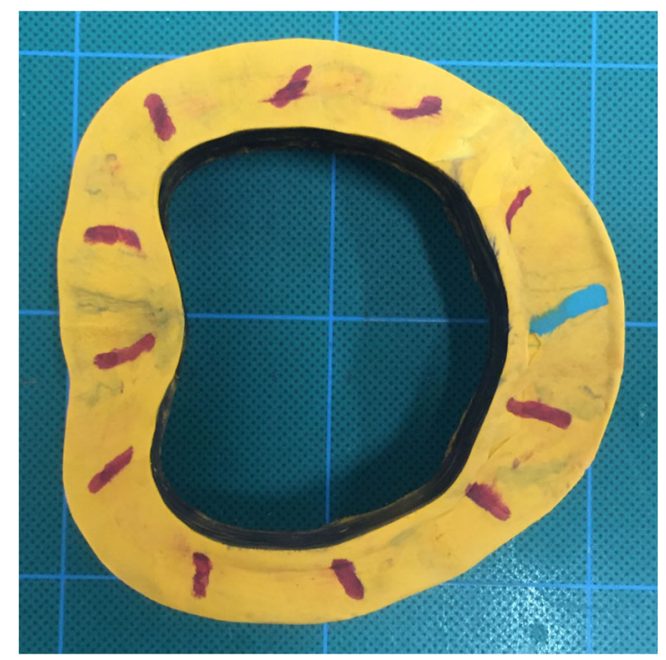

Fig. 28 Picture of final D ring produced using the propsed sensing and control strategy on a desktop-scale modelling clar RARR mill

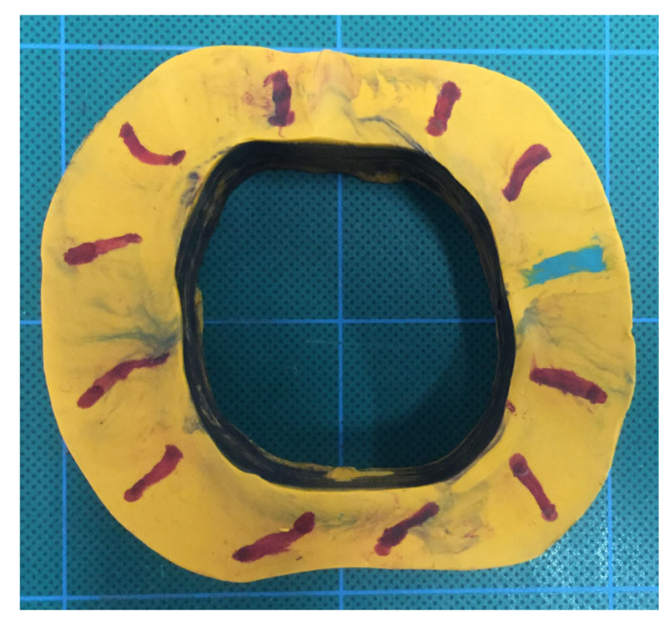

Fig. 29 Picture of final square ring produced using the propsed sensing and control strategy on a desktop-scale modelling clar RARR mill 


\section{Clay trials}

The trial shapes were also produced using the same control strategy using a desktop-scale RARR mill capable of rolling plasticine, which simulates the behaviour of metal at temperatures greater than half of the melting temperature [1]. In these real-world trials there were additional sources of error in the rolling process, primarily from underpowered actuators and a lack of machine stiffness.

The resultant shapes achieved during these trials can be seen in Figs. 28 and 29. Given that it is hard to separate the effects of the control strategy from the sources of error in the machine operation itself, we will not go into a detailed analysis of the dimensions of the final ring shapes. However, these results clearly demonstrate that it is possible to roll materials on real RARR hardware using the sensing and control strategy detailed in this paper.

\section{Conclusions and future research opportunities}

This work has described in detail a sensing and control strategy for adapting the conventional radial-axial ring rolling process to produce rings with variable wall thickness and variable curvature. Previous work has described the processes in isolation, but this is the first time that their combination has been shown to produce rings with a wide range of shapes. This is shown through numerical simulations and clay trials for two different target rings.

Using open-loop normalised tool path generation for the targeted shapes as a function of ring volume fraction permits the comparison of the evolving deforming body of the ring with the reference shape. This comparison is fundamental to the forming process and could be built upon further to automatically calculate the tool paths online using constrained feedback control. The control algorithm can be further improved through the development of better process models, or, if computational power permitted, through the use of other control strategies such as Model Predictive Control. Additional flexibility in the process can be achieved by incorporating simultaneous control of a variable axial height too, which will require additional sensing as well.

The development of a sensing and control system for production of rings with variable thickness and curvature using conventional RARR hardware leads to a major increase in the range of allowable ring geometry. This is of great importance to modern industry, as a wider range of products may potentially be produced with the benefits of RARR, such as efficient material usage and high material strength.
Compliance with Ethical Standards

Conflict of interests The authors declare that they have no conflict of interest.

Open Access This article is distributed under the terms of the Creative Commons Attribution 4.0 International License (http:// creativecommons.org/licenses/by/4.0/), which permits unrestricted use, distribution, and reproduction in any medium, provided you give appropriate credit to the original author(s) and the source, provide a link to the Creative Commons license, and indicate if changes were made.

Publisher's note Springer Nature remains neutral with regard to jurisdictional claims in published maps and institutional affiliations.

\section{References}

1. Aku S, Slater R, Johnson W (1967) The use of plasticine to simulate the dynamic compression of prismatic blocks of hot metal. Int J Mech Sci 9(8):495-500

2. Allwood J, Tekkaya A, Stanistreet T (2005) The development of ring rolling technology. Steel Res Int 76(2-3):111-120

3. Allwood J, Tekkaya A, Stanistreet T (2005) The development of ring rolling technology - part 2: Investigation of process behaviour and production equipment. Steel Res Int 76(7):491-507

4. Arthington M, Cleaver C, Allwood J, Duncan S (2015) Measurement and control of variable geometry during ring rolling. In: IEEE Multi-conference on systems and control, Sydney, pp 1448-1454

5. Arthington M, Cleaver C, Huang J, Duncan S (2016) Curvature control in radial-axial ring rolling. IFAC-PapersOnLine 49(20):244-249

6. Arthington MR, Cleaver C, Allwood J, Duncan S (2014) Real-time measurement of ring-rolling geometry using low-cost hardware. In: 2014 UKACC International conference on control, CONTROL 2014 - proceedings. IEEE, pp 603-608

7. Berti G, Quagliato L, Monti M (2015) Set-up of radial-axial ringrolling process: Process worksheet and ring geometry expansion prediction. Int J Mech Sci 99:58-71

8. Cleaver C, Allwood J (2017) Incremental profile ring rolling with axial and circumferential constraints. CIRP Ann 66(1):285-288

9. Cleaver C, Allwood J (2017) Incremental ring rolling to create conical profile rings. Procedia Eng 207:1248-1253

10. Davey K, Ward M (2002) The practicalities of ring rolling simulation for profiled rings. J Mater Process Technol 125126:619-625

11. Davis J, Semiatin S (1988) ASM Metals Handbook Volume 14: Forming and Forging. ASM International, Ohio

12. Epp J, Surm H, Kovac J, Hirsch T, Hoffmann F (2011) Interdependence of distortion and residual stress relaxation of cold-rolled bearing rings during heating. Metall and Mater Trans A 42(5):1205-1214

13. Jenkouk V, Hirt G, Franzke M, Zhang T (2012) Finite element analysis of the ring rolling process with integrated closed-loop control. CIRP Ann 61(1):267-270

14. Kluge A, Lee YH, Wiegels H, Kopp R (1994) Control of strain and temperature distribution in the ring rolling process. J Mater Process Technol 45(1-4):137-141

15. Meier H, Briselat J, Hammelmann R, Flick H (2010) Image Processing Methods for Online Measurement in Radial-Axial Ring Rolling. In: Proceedings of the 36th International MATADOR Conference. Springer, pp 355-358 
16. Meier H, Briselat J, Husmann T, Kreimeier D (2011) Online measurement of Radial-Axial rolled rings with an image processing system. In: Proceedings of the 18th International Forgemasters Meeting

17. Minton J, Brambley E (2017) Meta-analysis of curvature trends in asymmetric rolling. Procedia Eng 207:1355-1360

18. Minton JJ (2017) Mathematical modelling of asymmetrical metal rolling processes. $\mathrm{PhD}$ thesis, University of Cambridge

19. Parvizi A, Abrinia K (2014) A two dimensional upper bound analysis of the ring rolling process with experimental and fem verifications. Int J Mech Sci 79:176-181
20. Qian D, Zhang Z, Hua L (2013) An advanced manufacturing method for thick-wall and deep-groove ring-combined ring rolling. J Mater Process Technol 213(8):1258-1267

21. Wang C, Geijselaers H, Omerspahic E, Recina V, Van Den Boogaard A (2016) Influence of ring growth rate on damage development in hot ring rolling. J Mater Process Technol 227:268-280

22. Xu W, Yang X, Gong X, Zhou J (2012) A new mathematical model for predicting the diameter expansion of flat ring in radialaxial ring rolling. I J Adv Manuf Technol 60(9):913-921 